\title{
Global Well-Posedness for the $d$-Dimensional Magnetic Bénard Problem without Thermal Diffusion
}

\author{
Yinhong Cao \\ School of Mathematics and Information Science, Henan Polytechnic University, Jiaozuo, Henan 454100, China \\ Correspondence should be addressed to Yinhong Cao; caoyh@hpu.edu.cn
}

Received 19 March 2021; Accepted 11 May 2021; Published 4 June 2021

Academic Editor: Heng Liu

Copyright (c) 2021 Yinhong Cao. This is an open access article distributed under the Creative Commons Attribution License, which permits unrestricted use, distribution, and reproduction in any medium, provided the original work is properly cited.

This paper focuses on the global existence of strong solutions to the magnetic Bénard problem with fractional dissipation and without thermal diffusion in $\mathbb{R}^{d}$ with $d \geq 3$. By using the energy method and the regularization of generalized heat operators, we obtain the global regularity for this model under minimal amount dissipation.

\section{Introduction}

Consider the global well-posedness problem to the $d$-dimensional $(d D)$ magnetic Bénard problem

$$
\begin{cases}\partial_{t} u+u \cdot \nabla u+\mu \Lambda^{2 \alpha} u=-\nabla p+b \cdot \nabla b+\theta \mathbf{e}_{d}, & x \in \mathbb{R}^{d}, t>0, \\ \partial_{t} b+u \cdot \nabla b+v \Lambda^{2 \beta} b=b \cdot \nabla u, & x \in \mathbb{R}^{d}, t>0, \\ \partial_{t} \theta+u \cdot \nabla \theta=u \cdot \mathbf{e}_{d}, & x \in \mathbb{R}^{d}, t>0, \\ \nabla \cdot u=0, \nabla \cdot b=0, & x \in \mathbb{R}^{d}, t>0, \\ u(x, 0)=u_{0}(x), b(x, 0)=b_{0}(x), \theta(x, 0)=\theta_{0}(x), & x \in \mathbb{R}^{d},\end{cases}
$$

where $u(x, t), b(x, t), \theta(x, t)$, and $p(x, t)$ denote the velocity field, the magnetic field, the temperature, and the pressure, respectively. $\mu \geq 0$ is kinematic viscosity and $\nu \geq 0$ is magnetic diffusion. $\theta \mathbf{e}_{d}$ and $u \cdot \mathbf{e}_{d}$ model the acting of the buoyancy force on fluid motion and the Rayleigh-Bénard convection in a heated inviscid fluid, respectively. The parameters $\alpha$ and $\beta$ are nonnegative, and $\Lambda^{r} f$ with $r \geq 0$ is defined via $\overline{\Lambda^{r} f}(\xi)=|\xi|^{r} \widehat{f}(\xi)$. The magnetic Bénard problem can be used to model the behavior of the thermal instability under the influence of the magnetic field. One can refer to [1-7] for more physical background.
The global regularity problem of the magnetic Bénard problem (1) has caught much attention. For the 2D case, Zhou et al. in [8] obtained the global regularity for the case $\alpha=\beta=1$. When $\alpha=2$ and $\nu=0$, the global existence and uniqueness of strong solutions were established by Yamazaki in [9]. Recently, Shang in [10] showed the global wellposedness of solutions for the case $\alpha=1 / 2$ and $\beta=1$. Compared to the magnitude results on the $2 \mathrm{D}$ case, it appears that there are only few global regularity results on $d D$ $(d \geq 3)$ magnetic Bénard problem (1).

This paper focuses its attention on the global regularity problem of (1) with minimal amount dissipation. More 
precisely, we are able to establish the following global regularity result.

Theorem 1. Consider (1) with $\mu>0$ and $\nu>0$. Suppose that $\left(u_{0}, b_{0}, \theta_{0}\right) \in H^{s}\left(\mathbb{R}^{d}\right)$ with $s>1+d / 2, \nabla \cdot u_{0}=0$, and $\nabla \cdot b_{0}=0$. Assume also that

$$
\alpha \geq \frac{1}{2}+\frac{d}{4}, \alpha+\beta \geq 1+\frac{d}{2} .
$$

Then, (1) has a unique global strong solution $(u, b, \theta)$ satisfying, for any $T>0$,

$$
(u, b, \theta) \in L^{\infty}\left(0, T ; H^{s}\left(\mathbb{R}^{d}\right)\right), u \in L^{2}\left(0, T ; H^{s+\alpha}\left(\mathbb{R}^{d}\right)\right), w \in L^{2}\left(0, T ; H^{s+\beta}\left(\mathbb{R}^{d}\right)\right)
$$

In the subsequent section, we prove Theorem 1. Moreover, the definitions of the Besov spaces are provided in the appendix. We shall separately write $L^{p}=L^{p}\left(\mathbb{R}^{d}\right)$, $H^{s}=H^{s}\left(\mathbb{R}^{d}\right)$, and $\int=\int_{\mathbb{R}^{d}}$ for notational convenience.

\section{Proof of Theorem 1}

This section proves Theorem 1 . The key step is to establish global a priori $H^{s}$-bound for $(u, b, \theta)$. More specifically, we shall establish the following result.

Proposition 1. Consider (1) with $\mu>0$ and $\nu>0$. Suppose that $\left(u_{0}, b_{0}, \theta_{0}\right) \in H^{s}\left(\mathbb{R}^{d}\right) \quad$ with $s>1+d / 2$ and $\nabla \cdot u_{0}=\nabla \cdot b_{0}=0$, and

$$
\alpha \geq \frac{1}{2}+\frac{d}{4}, \alpha+\beta \geq 1+\frac{d}{2} .
$$

Then, the corresponding solution of (1) is globally bounded in $H^{s}\left(\mathbb{R}^{d}\right)$.

We only prove Proposition 1 for the case $\alpha+\beta=1+d / 2$. In fact, the case $\alpha+\beta>1+d / 2$ is even simpler.

2.1. Preparations. To prove the main theorem, as preparations we give three lemmas in this section. The first contains two calculus inequalities.

Lemma 1 (see $[11,12])$. Let $s>0$. Let $1<r<\infty$ and $1 / r=$ $1 / p_{1}+1 / q_{1}=1 / p_{2}+1 / q_{2} \quad$ with $q_{1}, p_{2} \in(1, \infty)$ and $p_{1}, q_{2} \in[1, \infty]$. Then,

$$
\left\|\left[\Lambda^{s}, f\right] g\right\|_{L^{r}} \leq C\left(\|\nabla f\|_{L^{p_{1}}}\left\|\Lambda^{s-1} g\right\|_{L^{q_{1}}}+\left\|\Lambda^{s} f\right\|_{L^{p_{2}}}\|g\|_{L^{q_{2}}}\right),
$$

with $\left[\Lambda^{s}, f\right] g=\Lambda^{s}(f g)-f \Lambda^{s} g$ and

$$
\left\|\Lambda^{s}(f g)\right\|_{L^{r}} \leq C\left(\left\|\Lambda^{s} f\right\|_{L^{p_{1}}}\|g\|_{L^{q_{1}}}+\|f\|_{L^{p_{2}}}\left\|\Lambda^{s} g\right\|_{L^{q_{2}}}\right),
$$

where $C^{\prime} s$ are constants.

The second is the property of the generalized heat operator.

Lemma 2 (see, e.g., $[13,14]$ ). Let $\mathscr{A}=\left\{\xi \in \mathbb{R}^{d}: r_{1} \leq|\xi| \leq r_{2}\right\}$ with $0<r_{1}<r_{2}$ being constants. Then, there exist two positive constants $c$ and $C$ such that, for

$$
f \in L^{p}\left(\mathbb{R}^{d}\right), \operatorname{supp} \widehat{f} \subset \lambda \mathscr{A}, p \in[1, \infty],
$$

and we have, for $\alpha>0, t>0$, and $\lambda>0$,

$$
\left\|e^{-(-\Delta)^{\alpha} t} f\right\|_{L^{p}\left(\mathbb{R}^{d}\right)} \leq C e^{-c t \lambda^{2 \alpha}}\|f\|_{L^{p}\left(\mathbb{R}^{d}\right)} .
$$

The last is the following logarithmic type interpolation inequality.

Lemma 3 (see $[14,15]$ ). Let $s>1+d / 2$ and $q \in(1, \infty)$. Then, there exists $C>0$ such that

$$
\|\nabla f\|_{L^{\infty}} \leq C\left(1+\|f\|_{L^{2}}+\left\|\Lambda^{d / q} \nabla f\right\|_{L^{q}} \log \left(e+\left\|\Lambda^{s} f\right\|_{L^{2}}\right)\right) .
$$

2.2. Global $H^{s}$ Bound. This section gives the proof of Proposition 1 . We first prove the following global $L^{2}$-bound.

Lemma 4. For any $t>0$, the solution $(u, b, \theta)$ of (1) obeys

$$
\|u\|_{L^{2}}^{2}+\|b\|_{L^{2}}^{2}+\|\theta\|_{L^{2}}^{2}+\int_{0}^{t}\left\|\Lambda^{\alpha} u(\tau)\right\|_{L^{2}}^{2} \mathrm{~d} \tau+\int_{0}^{t}\left\|\Lambda^{\beta} b(\tau)\right\|_{L^{2}}^{2} \mathrm{~d} \tau \leq C .
$$

Proof. Taking the $L^{2}$-inner product of (1) with $(u, b, \theta)$, together with the Young inequality, we obtain

$$
\begin{aligned}
& \frac{1}{2} \frac{\mathrm{d}}{\mathrm{d} t}\left(\|u\|_{L^{2}}^{2}+\|b\|_{L^{2}}^{2}+\|\theta\|_{L^{2}}^{2}\right)+\mu\left\|\Lambda^{\alpha} u\right\|_{L^{2}}^{2}+\nu\left\|\Lambda^{\beta} b\right\|_{L^{2}}^{2} \\
& =\int \theta \mathbf{e}_{d} \cdot u+\int u \cdot \mathbf{e}_{d} \theta \\
& \leq\|u\|_{L^{2}}^{2}+\|\theta\|_{L^{2}}^{2} .
\end{aligned}
$$

Then, (10) follows from this and Gronwall's inequality. The second one is a better regularity for $b$.

Lemma 5. Let $(u, b, \theta)$ be the solution of (1). Then, for all $t>0$ and $0<\sigma<\alpha-1$,

$$
\left\|\Lambda^{\rho} b(t)\right\|_{L^{2}}^{2}+\int_{0}^{t}\left\|\Lambda^{\beta+\sigma} b(\tau)\right\|_{L^{2}}^{2} \mathrm{~d} \tau \leq C .
$$


Proof. Applying $\Lambda^{\sigma}$ to $(1.1)_{2}$ and dotting the result with $\Lambda^{\sigma} b$, we have

$$
\frac{1}{2} \frac{\mathrm{d}}{\mathrm{d} t}\left\|\Lambda^{\sigma} b\right\|_{L^{2}}^{2}+v\left\|\Lambda^{\beta+\sigma} b\right\|_{L^{2}}^{2}=\int \Lambda^{\sigma}(b \cdot \nabla u) \cdot \Lambda^{\sigma} b-\int \Lambda^{\sigma}(u \cdot \nabla b) \cdot \Lambda^{\sigma} b:=I_{1}+I_{2} .
$$

Applying Lemma 1, we arrive at

$$
\begin{aligned}
I_{1} & \leq C\left(\left\|\Lambda^{\sigma} b\right\|_{L^{2} d / d-2 \beta}\|\nabla u\|_{L^{d / \beta}}+\left\|\Lambda^{\sigma} \nabla u\right\|_{L^{2 d / d+2+2 \sigma-2 \alpha}}\|b\|_{L^{d / \alpha-1-\sigma}}\right)\left\|\Lambda^{\sigma} b\right\|_{L^{2}} \\
& \leq C\left\|\Lambda^{\beta+\sigma} b\right\|_{L^{2}}\left\|\Lambda^{\alpha} u\right\|_{L^{2}}\left\|\Lambda^{\sigma} b\right\|_{L^{2}} \\
& \leq \frac{\nu}{4}\left\|\Lambda^{\beta+\sigma} b\right\|_{L^{2}}^{2}+C\left\|\Lambda^{\alpha} u\right\|_{L^{2}}^{2}\left\|\Lambda^{\sigma} b\right\|_{L^{2}}^{2} .
\end{aligned}
$$

Note that

$$
I_{2}=-\int\left(\Lambda^{\sigma}(u \cdot \nabla b)-u \cdot \nabla \Lambda^{\sigma} b\right) \cdot \Lambda^{\sigma} b .
$$

Then, by again applying Lemma 1 , we find that $I_{2}$ obeys the same bound as $I_{1}$. Combing the above estimates up, together with (10), Gronwall's inequality yields (12).

The last preparation is stated as follows.

Lemma 6. Let $(u, b, \theta)$ be the solution of (1). Then, for all $t>0$ and $d / 2 \alpha-2<q<\infty$,

$$
\int_{0}^{t}\left\|\Lambda^{d / q} \omega(\tau)\right\|_{L^{q}} \mathrm{~d} \tau<\infty
$$

where $\omega=\nabla \times u$.

In addition, for all $2 \leq q<\infty$,

$$
\|\theta(t)\|_{L^{q}}<\infty \text {. }
$$

Proof. Taking curl on the both sides of $(1.1)_{1}$, we have

$$
\partial_{t} \omega+\mu(-\Delta)^{\alpha} \omega=\nabla \times(b \cdot \nabla b)-\nabla \times(u \cdot \nabla u)+\nabla \times\left(\theta \mathbf{e}_{d}\right),
$$

with $\omega=\nabla \times u$. Write (18) into the integral form

$$
\begin{aligned}
\omega(t)= & e^{-\mu(-\Delta)^{\alpha} t} \omega_{0}+\int_{0}^{t} e^{-\mu(-\Delta)^{\alpha}(t-\tau)} \nabla \times \nabla \cdot(b \otimes b)(\tau) \mathrm{d} \tau \\
& -\int_{0}^{t} e^{-\mu(-\Delta)^{\alpha}(t-\tau)} \nabla \times \nabla \cdot(u \otimes u)(\tau) \mathrm{d} \tau+\int_{0}^{t} e^{-\mu(-\Delta)^{\alpha}(t-\tau)} \nabla \times\left(\theta \mathbf{e}_{d}\right)(\tau) \mathrm{d} \tau
\end{aligned}
$$

We further localize it by applying $\Delta_{j}$ with $j \geq-1$ :

$$
\begin{aligned}
\Delta_{j} \omega(t)= & \Delta_{j} e^{-\mu(-\Delta)^{\alpha} t} \omega_{0}++\int_{0}^{t} \Delta_{j} e^{-\mu(-\Delta)^{\alpha}(t-\tau)} \nabla \times \nabla \cdot(b \otimes b)(\tau) \mathrm{d} \tau \\
& -\int_{0}^{t} \Delta_{j} e^{-\mu(-\Delta)^{\alpha}(t-\tau)} \nabla \times \nabla \cdot(u \otimes u)(\tau) \mathrm{d} \tau+\int_{0}^{t} \Delta_{j} e^{-\mu(-\Delta)^{\alpha}(t-\tau)} \nabla \times\left(\theta \mathbf{e}_{d}\right)(\tau) \mathrm{d} \tau .
\end{aligned}
$$

For $q \in(1, \infty)$, taking the $L^{q}$-norm to this equation, we derive that 


$$
\begin{aligned}
& \left\|\Delta_{j} \omega(t)\right\|_{L^{q}} \leq C e^{-c_{0} \mu t 2^{2 \alpha j}}\left\|\Delta_{j} \omega_{0}\right\|_{L^{q}}+C \int_{0}^{t} 2^{2 j} e^{-c_{0} \mu(t-\tau) 2^{2 \alpha j}}\left\|\Delta_{j}(b \otimes b)(\tau)\right\|_{L^{q}} \mathrm{~d} \tau \\
& +C \int_{0}^{t} 2^{2 j} e^{-c_{0} \mu(t-\tau) 2^{2 \alpha j}}\left\|\Delta_{j}(u \otimes u)(\tau)\right\|_{L^{q}} \mathrm{~d} \tau+C \int_{0}^{t} 2^{j} e^{-c_{0} \mu(t-\tau) 2^{2 \alpha j}}\left\|\Delta_{j} \theta(\tau)\right\|_{L^{q}} \mathrm{~d} \tau .
\end{aligned}
$$

Choose $\sigma$ such that

$$
0<\sigma<\alpha-1, \sigma+\beta=\frac{d}{2}\left(1-\frac{1}{q}\right)
$$

By Sobolev's inequality,

$$
\left\|\Delta_{j}(b \otimes b)(\tau)\right\|_{L^{q}} \leq\|b \otimes b(\tau)\|_{L^{q}} \leq\|b\|_{L^{2 q}}^{2} \leq C\left\|\Lambda^{\sigma+\beta} b\right\|_{L^{2}}^{2} .
$$

Similarly, we have

$$
\left\|\Delta_{j}(u \otimes u)(\tau)\right\|_{L^{q}} \leq C\left(\|u\|_{L^{2}}^{2}+\left\|\Lambda^{2 \alpha-1} u\right\|_{L^{2}}^{2}\right)=C\|u\|_{H^{2 \alpha-1}}^{2} .
$$

We multiply the second equation in (1) by $\theta|\theta|^{q-2}$ and integrate the result in space domain to obtain

$$
\frac{1}{q} \frac{d}{d t}\|\theta\|_{L^{q}}^{q} \leq u \cdot \mathbf{e}_{d} \theta|\theta|^{q-2} \leq\|u\|_{L^{q}}\|\theta\|_{L^{q}}^{q-1}
$$

which yields

$$
\|\theta\|_{L^{q}} \leq\left\|\theta_{0}\right\|_{L^{q}}+\int_{0}^{t}\|u(\tau)\|_{L^{q}} \mathrm{~d} \tau \leq C+C \int_{0}^{t}\|u(\tau)\|_{H^{2 \alpha-1}} \mathrm{~d} \tau .
$$

Note that $\left\|\Delta_{j} \theta\right\|_{L^{q}} \leq\|\theta\|_{L^{q}}$; then, inserting (23)-(26) into (21), we derive that

$$
\begin{aligned}
& \left\|\Delta_{j} \omega(t)\right\|_{L^{q}} \leq C e^{-c_{0} \mu t 2^{2 \alpha j}}\left\|\Delta_{j} \omega_{0}\right\|_{L^{q}}+C \int_{0}^{t} 2^{2 j} e^{-c_{0} \mu(t-\tau) 2^{2 \alpha j}}\left\|\Lambda^{\sigma+\beta} b(\tau)\right\|_{L^{2}}^{2} \mathrm{~d} \tau \\
& +C \int_{0}^{t} 2^{2 j} e^{-c_{0} \mu(t-\tau) 2^{2 \alpha j}}\|u(\tau)\|_{H^{2 \alpha-1}}^{2} \mathrm{~d} \tau+C \int_{0}^{t} 2^{j e^{\left.-c_{0} \mu(t-\tau)\right)^{2 \alpha j}}\|u(\tau)\|_{H^{2 \alpha-1}} \mathrm{~d} \tau .}
\end{aligned}
$$

Integrating this in time, together with (12), we obtain

$$
\begin{aligned}
& 2^{(2 \alpha-2) j} \int_{0}^{t}\left\|\Delta_{j} \omega(\tau)\right\|_{L^{q}} \mathrm{~d} \tau \leq C 2^{-2 j}\left\|\Delta_{j} \omega_{0}\right\|_{L^{q}}+C \int_{0}^{t}\left\|\Lambda^{\sigma+\beta} b(\tau)\right\|_{L^{2}}^{2} \mathrm{~d} \tau+C \int_{0}^{t}\left(\|u(\tau)\|_{H^{2 \alpha-1}}^{2}+\|u(\tau)\|_{H^{2 \alpha-1}}\right) \mathrm{d} \tau \\
& \leq C+C \int_{0}^{t}\left(\|u(\tau)\|_{H^{2 \alpha-1}}^{2}+\|u(\tau)\|_{H^{2 \alpha-1}}\right) \mathrm{d} \tau .
\end{aligned}
$$

Next, we apply $\Lambda^{\alpha-1}$ to the first equation of (1) to obtain

$$
\begin{aligned}
& \frac{1}{2} \frac{d}{d t}\left\|\Lambda^{\alpha-1} u\right\|_{L^{2}}^{2}+\mu\left\|\Lambda^{2 \alpha-1} u\right\|_{L^{2}}^{2} \\
& =\int \Lambda^{\alpha-1}(b \cdot \nabla b) \cdot \Lambda^{\alpha-1} u-\int \Lambda^{\alpha-1}(u \cdot \nabla u) \cdot \Lambda^{\alpha-1} u+\int \Lambda^{\alpha-1}\left(\theta \mathbf{e}_{d}\right) \cdot \Lambda^{\alpha-1} u \\
& :=J_{1}+J_{2}+J_{3} .
\end{aligned}
$$

Using $\nabla \cdot b=0$, 


$$
\begin{aligned}
J_{1} & \leq C\left\|\Lambda^{-1} \nabla \cdot(b \otimes b)\right\|_{L^{2}}\left\|\Lambda^{2 \alpha-1} u\right\|_{L^{2}} \\
& \leq C\|b\|_{L^{4}}\left\|\Lambda^{2 \alpha-1} u\right\|_{L^{2}} \\
& \leq \frac{\mu}{8}\left\|\Lambda^{2 \alpha-1} u\right\|_{L^{2}}^{2}+C\left\|\Lambda^{\sigma_{0}+\beta} b\right\|_{L^{2}}^{2},
\end{aligned}
$$

where we have chosen $\sigma_{0}$ satisfying

$$
0<\sigma_{0}<\alpha-1, \sigma_{0}+\beta=\frac{d}{4}
$$

Similarly, we have

$$
\begin{aligned}
J_{2} & \leq C\left\|\Lambda^{-1} \nabla \cdot(u \otimes u)\right\|_{L^{2}}\left\|\Lambda^{2 \alpha-1} u\right\|_{L^{2}} \\
& \leq C\|u\|_{L^{4}}\left\|\Lambda^{2 \alpha-1} u\right\|_{L^{2}} \\
& \leq \frac{\mu}{8}\left\|\Lambda^{2 \alpha-1} u\right\|_{L^{2}}^{2}+C\left\|\Lambda^{\alpha} u\right\|_{H^{1}}^{2}, \\
J_{3} & \leq C\left\|\theta \mathbf{e}_{d}\right\|\left\|_{L^{2}}\right\| \Lambda^{2 \alpha-2} u \|_{L^{2}} \\
& \leq C\|\theta\|_{L^{2}}\|u\|_{L^{2}}^{1 / 2 \alpha-1}\left\|\Lambda^{2 \alpha-1} u\right\|_{L^{2}}^{2 \alpha-2 / 2 \alpha-1} \\
& \leq \frac{\mu}{8}\left\|\Lambda^{2 \alpha-1} u\right\|_{L^{2}}^{2}+C\left(\|u\|_{L^{2}}^{2}+\|\theta\|_{L^{2}}^{2}\right) .
\end{aligned}
$$

Inserting (30)-(33) into (29),

$$
\begin{aligned}
& \frac{\mathrm{d}}{\mathrm{d} t}\left\|\Lambda^{\alpha-1} u\right\|_{L^{2}}^{2}+\mu\left\|\Lambda^{2 \alpha-1} u\right\|_{L^{2}}^{2} \\
& \leq C\left(\left\|\Lambda^{\sigma_{0}+\beta} b\right\|_{L^{2}}^{2}+\left\|\Lambda^{\alpha} u\right\|_{H^{1}}^{2}+\|u\|_{L^{2}}^{2}+\|\theta\|_{L^{2}}^{2}\right) .
\end{aligned}
$$

Then, Gronwall's inequality and (10)-(12) yields

$$
\left\|\Lambda^{\alpha-1} u\right\|_{L^{2}}^{2}+\int_{0}^{t}\left\|\Lambda^{2 \alpha-1} u(\tau)\right\|_{L^{2}}^{2} \mathrm{~d} \tau \leq C .
$$

This together with (26) yields (17). Substituting (35) into (28), we obtain

$$
\sup _{j \geq-1} 2^{(2 \alpha-2) j} \int_{0}^{t}\left\|\Delta_{j} \omega\right\|_{L^{q}} \mathrm{~d} \tau<\infty .
$$

Thus, for $q>d / 2 \alpha-2$,

$$
\begin{aligned}
\left\|\Lambda^{d / q} \omega(\tau)\right\|_{L^{q}} \mathrm{~d} \tau & \leq C \int_{0}^{t}\|\omega(\tau)\|_{B_{q, 1}^{d / q}} \mathrm{~d} \tau \\
& =C \int_{0}^{t} \sum_{j \geq-1} 2^{d / q j}\left\|\Delta_{j} \omega(\tau)\right\|_{L^{q}} \mathrm{~d} \tau \\
& \leq C \int_{0}^{t} \sum_{j \geq-1} 2^{(d / q-(2 \alpha-2)) j} 2^{(2 \alpha-2) j}\left\|\Delta_{j} \omega(\tau)\right\|_{L^{q}} \mathrm{~d} \tau \\
& =C \sum_{j \geq-1} 2^{(d / q-(2 \alpha-2)) j} 2^{(2 \alpha-2) j} \int_{0}^{t}\left\|\Delta_{j} \omega(\tau)\right\|_{L^{q}} \mathrm{~d} \tau \\
& \leq C \sup _{j \geq-1} 2^{(2 \alpha-2) j} \int_{0}^{t}\left\|\Delta_{j} \omega(\tau)\right\|_{L^{q}} \mathrm{~d} \tau \sum_{j \geq-1} 2^{(d / q-(2 \alpha-2)) j} \\
& =C \sup _{j \geq-1} 2^{(2 \alpha-2) j} \int_{0}^{t}\left\|\Delta_{j} \omega(\tau)\right\|_{L^{q}} \mathrm{~d} \tau<\infty \\
& \quad \frac{1}{2} \frac{\mathrm{d}}{\mathrm{d} t}\left(\left\|\Lambda^{s} u\right\|_{L^{2}}^{2}+\left\|\Lambda^{s} b\right\|_{L^{2}}^{2}+\left\|\Lambda^{s} \theta\right\|_{L^{2}}^{2}\right)+\mu\left\|\Lambda^{s+\alpha} u\right\|_{L^{2}}^{2}+v\left\|\Lambda^{s+\beta} b\right\|_{L^{2}}^{2} \\
\text { disposal, we are ready to prove } & =K_{1}+K_{2}+K_{3}+K_{4}+K_{5}+K_{6}+K_{7}
\end{aligned}
$$

This is (16).

With Lemmas 4-6 at our disposal, we are ready to prove Proposition 1.

Proof. of Proposition 1. Applying $\Lambda^{s}$ to (1) and taking the $L^{2}$-inner product with $\left(\Lambda^{s} u, \Lambda^{s} b, \Lambda^{s} \theta\right)$ yields

where 


$$
\begin{aligned}
& K_{1}=-\int\left[\Lambda^{s}, u \cdot \nabla\right] u \cdot \Lambda^{s} u, \\
& K_{2}=\int \Lambda^{s}\left(\theta \mathbf{e}_{d}\right) \cdot \Lambda^{s} u, \\
& K_{3}=\int \Lambda^{s}(b \cdot \nabla b) \cdot \Lambda^{s} u, \\
& K_{4}=-\int\left[\Lambda^{s}, u \cdot \nabla\right] b \cdot \Lambda^{s} b, \\
& K_{5}=\int\left[\Lambda^{s}, b \cdot \nabla\right] u \cdot \Lambda^{s} b, \\
& K_{6}=-\int\left[\Lambda^{s}, u \cdot \nabla\right] \theta \cdot \Lambda^{s} \theta, \\
& K_{7}=\int \Lambda^{s}\left(u \cdot \mathbf{e}_{d}\right) \Lambda^{s} \theta .
\end{aligned}
$$

Taking advantage of Lemma 1,

$$
\left|K_{1}\right| \leq C\|\nabla u\|_{L^{\infty}}\left\|\Lambda^{s} u\right\|_{L^{2}}^{2} .
$$

By Young's inequality,

$$
\left|K_{2}+K_{7}\right| \leq\left\|\Lambda^{s} u\right\|_{L^{2}}^{2}+\left\|\Lambda^{s} \theta\right\|_{L^{2}}^{2} .
$$

Using $\nabla \cdot b=0$, we have

$$
\begin{aligned}
\left|K_{3}\right| & \leq C\left\|\Lambda^{s} b\right\|_{L^{2}}\|b\| \frac{d}{L^{\alpha-1}}\left\|\Lambda^{s+1} u\right\|_{L^{2} d / d+2-2 \alpha} \\
& \leq C\left\|\Lambda^{s} b\right\|_{L^{2}}\left\|\Lambda^{\beta} b\right\|_{L^{2}}\left\|\Lambda^{s+\alpha} u\right\|_{L^{2}} \\
& \leq \frac{\mu}{8}\left\|\Lambda^{s+\alpha} u\right\|_{L^{2}}^{2}+C\left\|\Lambda^{\beta} b\right\|_{L^{2}}^{2}\left\|\Lambda^{s} b\right\|_{L^{2}}^{2} .
\end{aligned}
$$

Similarly, by Lemma $1, K_{4}$ and $K_{5}$ are bounded by

$$
\begin{aligned}
& \left|K_{4}+K_{5}\right| \leq C\|\nabla u\|_{L^{\infty}}\left\|\Lambda^{s} b\right\|_{L^{2}}^{2}+C\left\|\Lambda^{s} b\right\|_{L^{2}}\|b\| \frac{d}{{ }_{L} \alpha-1}\left\|\Lambda^{s+1} u\right\|_{L^{2 d / d+2-2 \alpha}} \\
& \leq \frac{\mu}{8}\left\|\Lambda^{s+\alpha} u\right\|_{L^{2}}^{2}+C\|\nabla u\|_{L^{\infty}}\left\|\Lambda^{s} b\right\|_{L^{2}}^{2}+C\left\|\Lambda^{\beta} b\right\|_{L^{2}}^{2}\left\|\Lambda^{s} b\right\|_{L^{2}}^{2} .
\end{aligned}
$$

By Lemma 1,

$$
\begin{aligned}
\left|K_{6}\right| & \leq C\|\nabla u\|_{L^{\infty}}\left\|\Lambda^{s} \theta\right\|_{L^{2}}^{2}+C\left\|\Lambda^{s} \theta\right\|_{L^{2}}\left\|\Lambda^{1+s} u\right\|_{L^{2} d / d+2-2 \alpha}\|\theta\|_{L^{d / \alpha-1}} \\
& \leq C\|\nabla u\|_{L^{\infty}}\left\|\Lambda^{s} \theta\right\|_{L^{2}}^{2}+C\left\|\Lambda^{s} \theta\right\|_{L^{2}}\left\|\Lambda^{s+\alpha} u\right\|_{L^{2}}\|\theta\|_{L^{2 / \alpha-1}} \\
& \leq \frac{\mu}{8}\left\|\Lambda^{s+\alpha} u\right\|_{L^{2}}^{2}+C\|\nabla u\|_{L^{\infty}}\left\|\Lambda^{s} \theta\right\|_{L^{2}}^{2}+C\|\theta\|_{L^{d / \alpha-1}}^{2}\left\|\Lambda^{s} \theta\right\|_{L^{2}}^{2} .
\end{aligned}
$$

$$
\begin{aligned}
& \frac{\mathrm{d}}{\mathrm{d} t}\left(\left\|\Lambda^{s} u\right\|_{L^{2}}^{2}+\left\|\Lambda^{s} b\right\|_{L^{2}}^{2}+\left\|\Lambda^{s} \theta\right\|_{L^{2}}^{2}\right)+\mu\left\|\Lambda^{s+\alpha} u\right\|_{L^{2}}^{2}+\nu\left\|\Lambda^{s+\beta} b\right\|_{L^{2}}^{2} \\
& \leq C\left(1+\|\nabla u\|_{L^{\infty}}+\left\|\Lambda^{\beta} b\right\|_{L^{2}}^{2}+\|\theta\|^{2} \frac{d}{\alpha-1}\right)\left(\left\|\Lambda^{s} u\right\|_{L^{2}}^{2}+\left\|\Lambda^{s} b\right\|_{L^{2}}^{2}+\left\|\Lambda^{s} \theta\right\|_{L^{2}}^{2}\right) .
\end{aligned}
$$

We bound $\|\nabla u\|_{L^{\infty}}$ by Lemma 3. By Lemma 6, (10), and (17), we obtain

$$
\int_{0}^{t}\left\|\Lambda^{d / q} \omega(\tau)\right\|_{L^{q}} \mathrm{~d} \tau<\infty, \int_{0}^{t}\left\|\Lambda^{\beta} b\right\|_{L^{2}}^{2} \mathrm{~d} \tau<\infty, \int_{0}^{t}\|\theta\|_{L^{2 / \alpha-1}}^{2} \mathrm{~d} \tau<\infty .
$$


Then, the desired global $H^{s}$-bound follows from these bounds and Osgood's inequality. This completes the proof of Proposition 1.

\section{Appendix}

We recall the definitions of the Littlewood-Paley decomposition and Besov spaces in this appendix (see, e.g., $[13,16-19])$.

Let $\mathcal{S}$ be the usual Schwartz class and $\mathcal{S}^{\prime}$ be its dual. Write for each $j \in \mathbb{Z}$,

$$
A_{j}=\left\{\xi \in \mathbb{R}^{d}: 2^{j-1} \leq|\xi|<2^{j+1}\right\} .
$$

The Littlewood-Paley decomposition means that there exist functions $\left\{\Phi_{j}\right\}_{j \in \mathbb{Z}} \in \mathcal{S}$ such that

$$
\begin{aligned}
& \operatorname{supp} \widehat{\Phi}_{j} \subset A_{j}, \widehat{\Phi}_{j}(\xi)=\widehat{\Phi}_{0}\left(2^{-j} \xi\right) \text { or } \Phi_{j}(x)=2^{j d} \Phi_{0}\left(2^{j} x\right), \\
& \sum_{j=-\infty}^{\infty} \widehat{\Phi}_{j}(\xi)= \begin{cases}1, & \text { if } \xi \in \mathbb{R}^{d} \backslash\{0\}, \\
0, & \text { if } \xi=0 .\end{cases}
\end{aligned}
$$

Thus, for $\psi \in \mathcal{S}$, we have

$$
\sum_{j=-\infty}^{\infty} \widehat{\Phi}_{j}(\xi) \widehat{\psi}(\xi)=\widehat{\psi}(\xi) \quad \text { for } \xi \in \mathbb{R}^{d} \backslash\{0\} .
$$

Choose $\Psi \in \mathcal{S}$ such that

$$
\widehat{\Psi}(\xi)=1-\sum_{j=0}^{\infty} \widehat{\Phi}_{j}(\xi), \quad \xi \in \mathbb{R}^{d} .
$$

Then, for all $\psi \in \mathcal{S}$,

$$
\Psi * \psi+\sum_{j=0}^{\infty} \Phi_{j} * \psi=\psi,
$$

and hence

$$
\Psi * f+\sum_{j=0}^{\infty} \Phi_{j} * f=f,
$$

in $\mathcal{S}^{\prime}$ for any $f \in \mathcal{S}^{\prime}$. In addition, set

$$
\Delta_{j} f= \begin{cases}0, & \text { if } j \leq-2, \\ \Psi * f, & \text { if } j=-1, \\ \Phi_{j} * f, & \text { if } j=0,1,2, \ldots\end{cases}
$$

Definition A.1. The inhomogeneous Besov space $B_{p, q}^{s}$ with $s \in \mathbb{R}$ and $p, q \in[1, \infty]$ consists of $f \in \mathcal{S}^{\prime}$ satisfying

$$
\|f\|_{B_{p, q}^{s}} \equiv\left\|2^{j s}\right\| \Delta_{j} f\left\|_{L^{p}}\right\|_{l q}<\infty,
$$

where $\Delta_{j} f$ is as defined in (A.2).

An important tool in dealing with Fourier localized functions is the following Bernstein's inequality.

Proposition A.1. Let $\alpha \geq 0$. Let $1 \leq p \leq q \leq \infty$.

(1) If $f$ satisfies

$$
\operatorname{supp} \hat{f} \subset\left\{\xi \in \mathbb{R}^{d}:|\xi| \leq K 2^{j}\right\},
$$

for some integer $j$ and a constant $K>0$, then

$$
\left\|(-\Delta)^{\alpha} f\right\|_{L^{q}\left(\mathbb{R}^{d}\right)} \leq C_{1} 2^{2 \alpha j+j d(1 / p-1 / q)}\|f\|_{L^{p}\left(\mathbb{R}^{d}\right)} .
$$

(2) If $f$ satisfies

$$
\operatorname{supp} \hat{f} \subset\left\{\xi \in \mathbb{R}^{d}: K_{1} 2^{j} \leq|\xi| \leq K_{2} 2^{j}\right\},
$$

for some integer $j$ and constants $0<K_{1} \leq K_{2}$, then

$$
C_{1} 2^{2 \alpha j}\|f\|_{L^{q}\left(\mathbb{R}^{d}\right)} \leq\left\|(-\Delta)^{\alpha} f\right\|_{L^{q}\left(\mathbb{R}^{d}\right)} \leq C_{2} 2^{2 \alpha j+j d(1 / p-1 / q)}\|f\|_{L^{p}\left(\mathbb{R}^{d}\right)},
$$

where $C_{1}$ and $C_{2}$ are constants depending on $\alpha, p$, and q only.

\section{Data Availability}

No data were used to support this study.

\section{Conflicts of Interest}

The author declares that there are no conflicts of interest.

\section{Acknowledgments}

This study was supported by the Fundamental Research Funds for Universities of Henan Province (grant no. NSFRF180317), the Foundation of the Education Department of Henan Province (grant no. 14B110014), and the
RFDP of Henan Polytechnic University (grant no. B2013054).

\section{References}

[1] S. Abe and S. Thurner, "Anomalous diffusion in view of Einsteins 1905 theory of Brownian motion," Physica A: Statistical Mechanics and Its Applications, vol. 356, no. 2-4, pp. 403-407, 2005.

[2] G. P. Galdi, "Nonlinear stability of the magnetic Bénard problem via a generalized energy method," Archive for Rational Mechanics and Analysis, vol. 87, no. 2, pp. 167-186, 1985.

[3] M. Jara, "Nonequilibrium scaling limit for a tagged particle in the simple exclusion process with long jumps," Communications on Pure and Applied Mathematics, vol. 62, no. 2, pp. 198-214, 2009. 
[4] A. Mellet, S. Mischler, and C. Mouhot, "Fractional diffusion limit for collisional kinetic equations," Archive for Rational Mechanics and Analysis, vol. 199, no. 2, pp. 493-525, 2011.

[5] G. Mulone and S. Rionero, "Necessary and sufficient conditions for nonlinear stability in the magnetic Bénard problem," Archive for Rational Mechanics and Analysis, vol. 166, no. 3, pp. 197-218, 2003.

[6] Y. Zhou, H. Wang, and H. Liu, "Generalized function projective synchronization of incommensurate fractional-order chaotic systems with inputs saturation," International Journal of Fuzzy Systems, vol. 21, no. 3, pp. 823-836, 2019.

[7] Z. Han, S. Li, and H. Liu, "Composite learning sliding mode synchronization of chaotic fractional-order neural networks," Journal of Advanced Research, vol. 25, pp. 87-96, 2020.

[8] Y. Zhou, J. Fan, and G. Nakamura, "Global Cauchy problem for a $2 \mathrm{D}$ magnetic Bénard problem with zero thermal conductivity," Applied Mathematics Letters, vol. 26, no. 6, pp. 627-630, 2013.

[9] K. Yamazaki, "Global regularity of generalized magnetic Benard problem," Mathematical Methods in the Applied Sciences, vol. 40, pp. 2013-2033, 2017.

[10] H. Shang, "Global regularity results for the 2D magnetic Bénard problem with fractional dissipation," Journal of Mathematical Fluid Mechanics, vol. 21, no. 3, 2019.

[11] T. Kato and G. Ponce, "Commutator estimates and the Euler and the Navier-Stokes equations," Communications on Pure and Applied Mathematics, vol. 41, no. 7, pp. 891-907, 1988.

[12] C. E. Kenig, G. Ponce, and L. Vega, "Well-posedness of the initial value problem for the Korteweg-de Vries equation," Journal of the American Mathematical Society, vol. 4, no. 2, p. 323, 1991.

[13] H. Bahouri, J.-Y. Chemin, and R. Danchin, Fourier Analysis and Nonlinear Partial Differential Equations, Springer, Berlin, Germany, 2011.

[14] H. Shang and J. Wu, "Global regularity for 2D fractional magneto-micropolar equations," Mathematische Zeitschrift, vol. 297, pp. 775-802, 2021.

[15] H. Shang and M. Li, "Global regularity for d-Dimensional micropolar equations with fractional dissipation," Applicable Analysis, vol. 98, no. 9, pp. 1567-1580, 2019.

[16] J. Bergh and J. Löfström, Interpolation Spaces, an Introduction, Springer-Verlag, Berlin-Heidelberg-New York, 1976.

[17] C. Miao, J. Wu, and Z. Zhang, Littlewood-Paley Theory and its Applications in Partial Differential Equations of Fluid Dynamics, Science Press, Beijing, China, (in Chinese), 2012.

[18] T. Runst and W. Sickel, Sobolev Spaces of Fractional Order, Nemytskij Operators and Nonlinear Partial Differential Equations, Walter de Gruyter, New York, NY, USA, 1996.

[19] H. Triebel, Theory of Function Spaces II, Birkhauser Verlag, Basel, Switzerland, 1992. 